\title{
Infertility Following Pelvic Inflammatory Disease
}

\author{
A.J. Pavletic, ${ }^{1 *}$ P. Wölner-Hanssen, ${ }^{2}$ J. Paavonen, ${ }^{3}$ S.E. Hawes, ${ }^{4}$ and \\ D.A. Eschenbach 5 \\ ${ }^{1}$ Department of Family Medicine, University of Nebraska Medical Center, Omaha, NE \\ ${ }^{2}$ Department of Obstetrics and Gynecology, Lund University, Lund, Sweden \\ ${ }^{3}$ Department of Obstetrics and Gynecology, University of Helsinki, Helsinki, Finland \\ ${ }^{4}$ Department of Obstetrics and Gynecology, University of Washington, Seattle, WA \\ ${ }^{5}$ Department of Medicine, University of Washington, Seattle, WA
}

\begin{abstract}
Objective: To assess the frequency of infertility after pelvic inflammatory disease (PID) and factors important in postinfectious tubal damage in an urban population at high risk for sexually transmitted diseases.

Methods: From a cohort of 213 women with PID documented by laparoscopy and/or endometrial biopsy, 58 women ( $27 \%$ of the initial cohort) were interviewed by phone 2 to 9 years after an index episode of PID. Data regarding the initial history, physical examination, microbiology, laparoscopic, and serologic findings, and data concerning interval contraception, subsequent pregnancy, subsequent infection, and chronic pelvic pain were compared among those with and without infertility at follow up.

Results: Nineteen ( $40 \%$ ) of the 48 women not using contraception were involuntarily infertile after the index episode of PID. Compared with those who had an interval pregnancy, infertile women were older $(P=0.02)$, more likely to have a history of infertility prior to the index episode of PID ( $P=0.001)$, and were more likely to have occluded or partially occluded fallopian tubes $(P$ $=0.03)$, peritubal adhesions $(P=0.007)$, or perihepatic adhesions $(P=0.02)$ seen by laparoscopy performed during the index episode. Surprisingly, recovery of Chlamydia trachomatis was negatively related to infertility $(P=0.001)$, although a similar proportion of both groups had chlamydia immunoglobulin $M$ antibody (40\% vs. $31 \%$ ). Chlamydia heat shock protein was weakly related to infertility $(P=0.08)$. The isolation of Neisseria gonorrhoeae was not significantly different between groups (53\% vs. 57\%).

Conclusions: The high rate of postinfection infertility found was probably related to a combination of tubal damage before and during the index episode of PID. Prevention of recurrent PID and better understanding of the pathophysiology of postinfection tubal damage (which may differ between chlamydia and gonorrhea) is needed to develop more effective strategies to reduce permanent tubal damage. Infect. Dis. Obstet. Gynecol. 7:145-152, 1999.
\end{abstract}

KEY WORDS

PID; infertility; $C$. trachomatis; $N$. gonorrhoeae; perihepatitis; chlamydial heat shock protein

$\mathrm{T}$ he link between acute pelvic inflammatory disease (PID) and sequelae such as tubal factor infertility and ectopic pregnancy have been documented in large long-term prospective Swedish studies. ${ }^{1-3}$ The infertility rate after an episode of
PID most closely correlates with the severity of tubal damage seen at laparoscopy during the acute episode of PID. Infertility occurred in $3 \%$ of patients with mild tubal damage, $13 \%$ of patients with moderate tubal damage, and $29 \%$ of patients

Grant sponsor: Division of Allergy and Infectious Disease, National Institutes of Health; Grant numbers: AI 24756 and AI 33118.

*Correspondence to: Adriana J. Pavletic, M.D., University of Nebraska Medical Center, Department of Family Medicine, 983075 Nebraska Medical Center, Omaha, NE 68198-3075. 
with severe tubal damage observed laparoscopically. ${ }^{1}$ Infertility also increases dramatically with recurrent episodes of PID. Infertility occurred in $8 \%$ of patients with one episode, $20 \%$ of patients with two episodes, and $40 \%$ in those with three or more episodes of PID. ${ }^{2}$ Infertility was higher following nongonococcal PID (17\%) than gonococcal PID $(6 \%)^{1}$ and was also associated with age 24 years at the time of the index episode of PID. ${ }^{3}$

In the past two decades, the prevalence of $C h l a-$ mydia trachomatis has exceeded that of Neisseria gonorrhoeae in Scandinavian countries ${ }^{3}$ and in many areas of the United States. ${ }^{4}$ Numerous seroepidemiological studies, both in Europe and North America, have consistently documented a higher prevalence of $C$. trachomatis antibody in infertile patients with tubal infertility than in either infertile patients without tubal damage or fertile patients. ${ }^{5,6}$ Few reports are available on the relative role of $N$. gonorrhoeae and other microorganisms in tubal infertility. ${ }^{7,8}$ In one study, PID with $C$. trachomatis was associated with a higher rate of subsequent tubal infertility than PID with $N$. gonorrhoeae, ${ }^{9}$ but this finding has not been uniform. ${ }^{10,11}$

In this study, we obtained long term follow-up data from patients with previously documented acute PID. The aim of the study was to assess the frequency of long-term sequelae and examine epidemiologic, clinical, and microbiologic features of acute PID that might contribute to infertility, with an emphasis on the impact of $C$. trachomatis.

\section{SUBJECTS AND METHODS}

The study population was derived from an original group of 395 patients with suspected acute PID enrolled between June 1983 and June 1990 at Harborview Medical Center, Seattle, Washington. Of the 395 patients with suspected PID, 213 (54\%) had the diagnosis either visually documented by laparoscopy $(n=119)$ or by the presence of plasma cells on endometrial biopsy $(n=94)$. Minimal criteria used for the enrollment of patients with suspected PID into the study included lower abdominal pain of less than three weeks' duration, abnormal adnexal tenderness, and willingness to participate in the study. Patients were excluded if they were younger than 16 years or older than 50 years, agreed neither to endometrial biopsy nor to laparoscopy, had taken antibiotics in the previous 2 weeks, were allergic to certain antibiotics used in treatment trials, were pregnant, had undergone pelvic or abdominal surgery or termination of pregnancy within the previous 45 days, had severe underlying mental or physical disorders, or did not speak English. The criteria of acute salpingitis observed by laparoscopy were those outlined by Jacobson and Weström. ${ }^{12}$ The criteria of plasma cell endometritis have been published elsewhere. ${ }^{13}$ All patients signed a written consent approved by the University of Washington Human Subjects Committee for the study procedures including permission to gather long-term follow-up data. We were able to locate 58 patients (27\%) from 213 patients originally diagnosed with PID using hospital records, telephone directories, and registered letters to the last known address.

Patients with suspected PID had undergone standardized interviews on demographic characteristics; education level; medical, sexual, and contraceptive-use history; and present symptoms. Patients considered to have probable tubal infertility prior to the index episode of PID were those who had had unprotected intercourse for $\geq 12$ months with no resulting pregnancy. A standardized physical examination, including a pelvic examination, was performed. Cervical, endometrial, and, in patients undergoing laparoscopy, tubal cultures were collected. Isolation methods have been previously described. ${ }^{14}$ Blood was drawn and tested for chlamydia serological response and chlamydial heat shock protein-60 (Chsp-60). ${ }^{15}$ To be consistent with prior reports, an elevated Chsp-60 level was defined as an optical density (OD) $\geq 0.2$, with a higher level of $\mathrm{OD} \geq 0.5 .^{15} \mathrm{Chsp}-60$ antibody determinations were kindly performed by Roseanna Peeling, Ph.D., National Laboratories for Sexually Transmitted Disease, Winnipeg, Manitoba, Canada. Laparoscopy and/or endometrial biopsy were performed on all patients. Patients were treated with standard antibiotics regimens to inhibit both $N$. gonorrhoeae and $C$. trachomatis.

Between July 1991 and February 1992, attempts were made to locate all women in the cohort. 'The phone numbers and addresses of patients at the last clinic visit were obtained from computerized records of the Sexually Transmitted Disease Clinic and outpatient clinic and inpatient records of Harborview Medical Center. A standardized interview form was developed concerning interval contraception use, subsequent episodes of gonorrhea, chla- 
mydia, or PID, subsequent pregnancy, perception of infertility, ectopic pregnancy, and chronic pelvic pain. Repeated attempts were made to contact by phone 66 patients with currently available phone numbers. We contacted 49 patients and interviewed 48. In addition, registered letters were sent to 17 patients not initially contacted and to 53 women without current telephone numbers who attended these clinics since 1985 . Ten patients responded to the letter and were interviewed by telephone. The remaining women could not be contacted. The interview was administered by one of the authors (A.J.P.) who was not aware of the characteristics of the index PID episode at the time of the interview.

At the index episode of PID, the 58 patients interviewed and 155 patients not interviewed were similar in the following parameters: mean age, age at first intercourse, median number of lifetime partners, education level, contraceptive use, clinical characteristics, and microbiologic etiology. The only difference between groups was a higher proportion of nonwhite, predominantly black patients in the interviewed group (72\%) compared with the group not interviewed $(48 \%, P=0.02)$. A detailed description of the initial cohort group will be published elsewhere. On follow up, patients with more than 2 years of regular unprotected intercourse without resulting pregnancy were considered infertile. Chronic pelvic pain was defined as pelvic pain of at least two months' duration requiring medication and/or medical attention.

\section{Statistical Methods}

To test for associations with fertility status, comparisons for categorical characteristics (race, marital status, etc.) were made using Pearson's chi-squares. When expected cell frequencies were smaller than five, Fisher exact tests were employed. Continuous factors, such as age and tenderness score, were analyzed with Student's $t$ test. However, some factors, such as number of lifetime sexual partners and days of pain, have severe outlier values, and associations between these factors and fertility status were analyzed using nonparametric Wilcoxon two-sample rank sum tests. ${ }^{16}$ In all cases, $P$ values $\leq 0.05$ were considered statistically significant.

\section{RESULTS}

The 58 patients were interviewed 2 to 9 years (mean, 4.8 years) after the index PID episode. The mean follow-up time was similar in the fertile (4.8 years) and infertile ( 4.5 years) groups, $P=0.5$. Ten patients were considered voluntarily infertile (seven had bilateral tubal ligations, two used oral contraception continuously, and one patient had not been sexually active since the index episode of PID). These 10 patients were excluded from the assessment of infertility. Of the remaining $48 \mathrm{pa}-$ tients, the initial diagnosis of acute PID was made by laparoscopy and endometrial biopsy in 30 and by endometrial biopsy without laparoscopy in 18 patients. An inability to become pregnant after at least 2 years of regular unprotected intercourse occurred for 19 (40\%) of the 48 patients not using birth control, compared with 29 patients who did become pregnant ('Table 1). The mean age at the index episode of PID was more than 3 years greater in patients designated infertile compared with patients designated fertile at follow up $(P=0.02)$. Age $>25$ years at the index episode of PID was also associated with subsequent infertility $(P<0.001$, Table 1). Patients in the two groups were of similar ethnic background. None of the 48 patients were married.

The age of first intercourse and number of median lifetime partners were similar in the two groups. The infertile group had a mean of 3 more years of sexual activity than the fertile group, relative to the 3-years-older mean age of this group. A past history of gonorrhea and chlamydia was common in both groups. A past history of PID prior to the index episode of PID occurred in $37 \%$ of the infertile and $28 \%$ of the fertile group $(P=0.5)$. In the infertile group, two patients had a history of pelvic abscess and three patients had an ectopic pregnancy prior to the index episode of PID.

The number of patients with a prior pregnancy, parity $\geq 1$, and who had had a live-born child, was similar between groups. A minority of patients in each group used birth control when the index episode of PID developed. Oral contraception was used by $5 \%$ of the infertile and $14 \%$ of the fertile group, and barrier contraception by $16 \%$ of the infertile and $21 \%$ of the fertile group.

A diagnosis of probable infertility at the time of the index episode of PID was also associated with subsequent infertility. Probable infertility at the in dex episode of PID was present in $76 \%$ of those designated infertile and $23 \%$ of those with a pregnancy in the interval between the index PID and 
TABLE I. Demographic, sexual, and pregnancy histories and clinical findings in patients at time of the acute index episode of PID stratified into fertile and infertile groups at follow-up

\begin{tabular}{|c|c|c|c|}
\hline \multirow[b]{2}{*}{ Characteristic at index PID episode } & \multicolumn{2}{|c|}{ Follow-up group } & \multirow[b]{2}{*}{$P$ value } \\
\hline & $\begin{array}{c}\text { Infertile } \\
(\mathrm{N}=19)\end{array}$ & $\begin{array}{c}\text { Fertile } \\
(\mathrm{N}=29)\end{array}$ & \\
\hline \multicolumn{4}{|l|}{ Demographics } \\
\hline Mean age $( \pm S D)^{a}$ & $25.1 \pm 5.3$ & $21.9 \pm 4.0$ & 0.02 \\
\hline Age $>25$ years & $10(53 \%)$ & $3(10 \%)$ & 0.001 \\
\hline Nonwhite ethnicity & $14(74 \%)$ & $21(72 \%)$ & 0.9 \\
\hline Married & 0 & $0 / 28$ & - \\
\hline \multicolumn{4}{|l|}{ Sexual } \\
\hline Age at first intercourse & $14.7 \pm 1.8$ & $|4.4 \pm 2|$. & 0.6 \\
\hline Median no. of lifetime partners & 10 & 8 & 0.3 \\
\hline Mean years sexually active & $10.1 \pm 5.2$ & $7.4 \pm 5.0$ & 0.09 \\
\hline \multicolumn{4}{|l|}{ Past history } \\
\hline Gonorrhea & $9(47 \%)$ & $14(48 \%)$ & 0.9 \\
\hline Chlamydia & $5 / 18(28 \%)$ & $14(48 \%)$ & 0.2 \\
\hline PID & $7(37 \%)$ & $8(28 \%)$ & 0.5 \\
\hline Pelvic abscess & $2 / 18(11 \%)$ & 0 & 0.1 \\
\hline Ectopic pregnancy & $3 / 18(17 \%)$ & 0 & 0.2 \\
\hline \multicolumn{4}{|l|}{ Pregnancy } \\
\hline Prior pregnancy & $14(74 \%)$ & $21(72 \%)$ & 0.9 \\
\hline Parity $\geq 1$ & $8(42 \%)$ & $17(59 \%)$ & 0.3 \\
\hline Any live born child & $8 / 14(57 \%)$ & $|7 / 2|(8 \mid \%)$ & 0.2 \\
\hline No birth control & $14(74 \%)$ & $18(62 \%)$ & 0.4 \\
\hline Probably infertile & $13 / 17(76 \%)$ & $6 / 26(23 \%)$ & 0.001 \\
\hline \multicolumn{4}{|l|}{ Physical and laboratory findings } \\
\hline Median days of pain & 3 & 5 & 0.8 \\
\hline Mean tenderness score $( \pm S D)$ & $18.7 \pm 9.0$ & $13.8 \pm 7.3$ & 0.04 \\
\hline Temperature $>38^{\circ} \mathrm{C}$ & $4 / 18(22 \%)$ & $5 / 28(18 \%)$ & 0.7 \\
\hline White blood cell count $>10,800$ & $9 / 18(50 \%)$ & $12 / 28(43 \%)$ & 0.6 \\
\hline Erythrocyte sedimentation rate $>20$ & $9 / 18(50 \%)$ & $14 / 25(56 \%)$ & 0.7 \\
\hline Abscess & $0 / 14(0)$ & $1 / 16(6 \%)$ & 0.3 \\
\hline
\end{tabular}

aSD, standard deviation.

follow up ( $P=0.001)$. At the time of our follow up 2 to 9 years later, successful attempts to achieve pregnancy occurred in six (32\%) of 19 patients with a history of probable infertility prior to the index episode (see definition in Materials and Methods) and in $20(83 \%)$ of 24 patients without the definition of probable infertility at the time of index PID episode $(P=0.02)$. Thus, while probable infertility at the index episode was highly associated with subsequent infertility, there was a significant overlap of subsequent fertility and infertility between the group designated probably infertile and the group considered fertile at the index episode of PID.

Presenting clinical characteristics during the index PID episode (median days of pain and an elevated temperature, white blood cell count, or erythrocyte sedimentation rate) did not differ between groups designated infertile or fertile at follow up. Patients in the two groups were treated with similar antibiotic regimens. A cephalosporin and doxycycline was given to $76 \%$ of 17 infertile patients and $75 \%$ of 28 fertile patients $(P=1.0)$. The remainder of patients received tobramycin or gentamicin with clindamycin. Hospitalization occurred for $63 \%$ of the 19 infertile and $59 \%$ of the 29 fertile patients $(P=1.0)$.

Laparoscopy at the index episodes of acute PID revealed that the infertility group had more moderate to severe tubal damage, peritubal adhesions, and perihepatic adhesions than the group with a subsequent pregnancy (Table 2). Nine of the ten patients without peritubal adhesions at the time of acute PID became pregnant. Pus in the posterior cul-de-sac was not related to subsequent infertility.

$N$. gonorrhoeae was initially isolated from a similar proportion of the group with subsequent infertility $(53 \%)$ and fertility $(57 \%, P=0.8$, Table 3$) . G$. trachomatis was initially isolated from none of 19 patients with subsequent infertility and $50 \%$ of the 
TABLE 2. Comparison of laparoscopic findings at time of acute PID and subsequent infertility at follow up

\begin{tabular}{lrrl}
\hline & \multicolumn{2}{c}{ Follow-up group } & \\
\cline { 2 - 3 } & Infertile & Fertile & \\
Laparoscopic findings & $(\mathrm{N}=14)$ & $(\mathrm{N}=16)$ & P value \\
\hline Moderate to severe salpingitis & $11(79 \%)$ & $6(37 \%)$ & 0.03 \\
Peritubal adhesions & $13(93 \%)$ & $7(44 \%)$ & 0.007 \\
Pus in cul-de-sac/abdomen & $5(36 \%)$ & $6(38 \%)$ & 0.9 \\
Perihepatic adhesions & $7 / 11(64 \%)$ & $3(19 \%)$ & 0.02 \\
\hline
\end{tabular}

28 patients with subsequent fertility $(P=0.001$, Table 3). A patient was considered to have N. gonorrhoeae or $C$. trachomatis if the microbe was isolated from any site (cervix, endometria, or tube) $C$. trachomatis was initially recovered at a similar rate among patients with $N$. gonorrhoeae (31\% of 26) as without $N$. gonorrhoeae (29\% of 21). A slightly higher proportion of patients were culture-negative for both $N$. gonorrhoeae and $C$. trachomatis in the infertile (47\%) than fertile (21\%) group $(P=0.1) . N$. gonorrhoeae and $C$. trachomatis were less often recovered from the upper than the lower genital tract at the index episode of PID. $N$. gonorrhoeae was recovered from the endometrium in 14 women, from the fallopian tubes in three, and from the cul-desac in two. C. trachomatis was recovered from the endometrium in five patients, from the fallopian tubes in two, and from the cul-de-sac in one.

The prevalence of immunoglobulin $\mathrm{G}$ (IgG) antibody to $C$. trachomatis at the index episode of acute PID was high in both the infertile and fertile groups (Table 3). There was a weak trend in which infertile patients demonstrated an elevated IgG antibody titer to $C$. trachomatis $\geq 1: 16(P=0.09)$. The IgM antibody to $C$. trachomatis was present in a similar proportion of patients in both groups. The mean OD of Chsp-60 was similar in the groups. However, a positive OD of $\geq 0.2$ for Chsp- 60 was present in $79 \%$ of 14 infertile patients and $52 \%$ of 23 fertile patients $(P=0.1)$. An $\mathrm{OD} \geq 0.2$ has been previously used as the cut off for positivity. ${ }^{15}$ Using a higher $O D$ of $\geq 0.5$, which distinguished tubal damage in this population, ${ }^{17}$ Chsp-60 was present in $64 \%$ of the infertile and $34 \%$ of the fertile patients $(P=0.08)$.

A sizable proportion of both groups reported subsequent gonorrhea ( $28 \%$ vs. $18 \%, P=0.5$ ), chlamydia (33\% vs. $18 \%, P=0.3$ ) and PID (6\% vs. $18 \%$, $P=0.4)$ after the index episode of acute PID. A total of 58 intrauterine pregnancies (mean, two; range, one to five) occurred in the subset of 29 patients after the index episode of acute PID. Infertile patients were followed from 3 to 6 years after the acute PID. Three fertile patients achieved their post-PID pregnancies after more than 1 year of trying (one after 1.5 years and two after 2 years). Another fertile patient had a normal pregnancy and delivery followed by two ectopic pregnancies in the same tube. Four years earlier she had severe salpingitis associated with both $N$. gonorrhoeae and $C$. trachomatis and reported no subsequent infections. Only three of $58(5.1 \%)$ patients reported a history of chronic pelvic pain. In one infertile patient, chronic pelvic pain resulted in a laparotomy after the index episode of PID where adhesions were lysed and a hydrosalpinx was removed. She was the only patient who reported chronic pelvic pain related to the index episode of PID. The other two patients (one in each of the fertile and infertile groups) had chronic pelvic pain before the index episode of PID.

\section{DISCUSSION}

This is the second long-term follow-up study in the United States after laparoscopic confirmation of acute PID. ${ }^{18}$ In the first report, $56 \%$ of 18 patients were involuntarily infertile after 1 year. ${ }^{18}$ The $40 \%$ rate of infertility in this population was identical to the $40 \%$ rate of infertility among a similar population in San Francisco, where PID was diagnosed by clinical criteria. ${ }^{19}$ A history of PID prior to the index episode of PID was also present in about $40 \%$ of both this and the San Francisco population. ${ }^{19}$ These infertility rates are higher than those in Weström's data, in which an $11.4 \%$ tubal-factor infertility rate was reported based on follow up of 1,732 Swedish women over a 25 -year period. ${ }^{2}$ A high rate of PID prior to the index episode contributed to a high infertility rate in our population. Nine infertile women appeared to develop infertility as a result of the index episode of PID. The remaining 10 women who were unable to become pregnant for 2 or more years after the index episode of acute PID also experienced difficulty becoming pregnant before the index episode of PID. Weström found an infertility rate of $20 \%$ in women with two episodes and $40 \%$ in women with three or more episodes of PID. ${ }^{2}$ Too few women were included in our study to prospectively examine the effect of multiple epi- 
TABLE 3. Initial isolation of $N$. gonorrhoeae and $C$. trachomatis and initial chlamydial serology at the time of acute PID stratified by infertility at follow-up

\begin{tabular}{|c|c|c|c|}
\hline & $\begin{array}{c}\text { Infertile } \\
(N=19)\end{array}$ & $\begin{array}{c}\text { Fertile } \\
(\mathrm{N}=\mathbf{2 8})\end{array}$ & $P$ value \\
\hline \multicolumn{4}{|l|}{ Isolation at index PID episode } \\
\hline N. gonorrhoeae only & $10(53 \%)$ & $8(29 \%)$ & 0.2 \\
\hline C. trachomatis only & $0(0 \%)$ & $6(21 \%)$ & 0.07 \\
\hline Both N. gonorrhoeae and C. trachomatis & $0(0 \%)$ & $8(29 \%)$ & 0.01 \\
\hline Neither microorganism & $9(47 \%)$ & $6(21 \%)$ & 0.1 \\
\hline Total N. gonorrhoeae & $10(53 \%)$ & $16(57 \%)$ & 0.8 \\
\hline Total C. trachomatis & $0(0 \%)$ & $14(50 \%)$ & $<0.001$ \\
\hline \multicolumn{4}{|l|}{ Serology at index PID episode } \\
\hline \multicolumn{4}{|l|}{ C. trachomatis lgG } \\
\hline$\leq 1: 8$ & $0 / 14(0 \%)$ & $5 / 28(18 \%)$ & \\
\hline $1: 16-1: 32$ & $8 / 14(57 \%)$ & $8 / 28(29 \%)$ & 0.09 \\
\hline$\geq 1: 64$ & $6 / 14(43 \%)$ & $15 / 28(54 \%)$ & \\
\hline \multicolumn{4}{|l|}{$\lg M$} \\
\hline Negative & $9 / 15(60 \%)$ & $18 / 26(69 \%)$ & \\
\hline$\leq 1: 8$ & $5 / 15(33 \%)$ & $6 / 26(23 \%)$ & 0.8 \\
\hline$\geq 1: 16$ & $1 / 15(7 \%)$ & $2 / 26(8 \%)$ & \\
\hline \multicolumn{4}{|l|}{ Chsp-60 } \\
\hline Mean OD & $0.7 \pm 0.5$ & $0.5 \pm 0.6$ & 0.4 \\
\hline$O D>0.2$ & $\mathrm{II} / 14$ (79\%) & $\mathrm{II} / 23(48 \%)$ & 0.1 \\
\hline$O D>0.5$ & $9 / 14(64 \%)$ & $8 / 23(35 \%)$ & 0.08 \\
\hline
\end{tabular}

sodes of PID on infertility, but these data support Weström's finding of an impact of multiple episodes of PID on infertility.

The correlation between laparoscopic findings at the index episode and subsequent infertility are consistent with previously published results that also concluded that severity of tubal damage at the index episode of acute PID predicts subsequent infertility. ${ }^{1,2}$ In contrast, the absence of peritubal adhesions at the time of acute PID was the laparoscopic finding most likely to be predictive of subsequent fertility. In another prior report, adhesions observed during the acute episode of PID at the index laparoscopy inevitably remained at a secondlook laparoscopy 3 to 5 weeks later, and fallopian tubes that appeared patent at initial evaluation remained patent as determined by chromotubation at follow up. ${ }^{20}$

In prior reports, patients with nongonococcal PID appear to have more infertility than those with gonococcal PID, ${ }^{1}$ even when adequate treatment is given to both groups. ${ }^{9}$ As expected, the prevalence of serum antibodies to both $N$. gonorrhoeae and $C$. trachomatis is elevated in infertile women with tubal disease compared with infertile women with normal tubes. ${ }^{7,8}$ C. trachomatis antibody is frequently found in infertile women, ${ }^{5,6}$ but prospective studies are scarce and contradictory as to whether women with $C$. trachomatis PID have dis- proportionate rates of infertility. Svensson reported that, while fallopian tubes were more severely damaged during the acute episode of PID among patients with $C$. trachomatis than those with $N$. gonorrhoeae, subsequent infertility rates were similar in each etiologic group. ${ }^{10}$ Only patients with one PID episode were analyzed to avoid the confounding effect of multiple infections. A second report found that cervical $G$. trachomatis isolation was not associated with increased severity of tubal damage in 64 patients with acute PID assessed by laparoscopy. ${ }^{21}$

These findings contrast with a Canadian report in which infertility occurred significantly more often with nongonococcal (seven of 13 cases) than gonococcal (zero of 10 cases) PID. ${ }^{9}$ Four of the seven infertile patients initially had a tubo-ovarian abscess, and three had culture (one patient) or serologic (two patients) evidence of $C$. trachomatis infection with the acute episode of PID. ${ }^{9}$ C. trachomatis was particularly related to infertility in a multivariate analysis of 43 Finnish women with PID in which serum chlamydial antibody was most likely to predict abnormal hysterosalpingogram (HSG) findings. ${ }^{11}$ However, an abnormal HSG after the episode of PID was not related to the isolation of $C$. trachomatis at the time of acute PID, ${ }^{11}$ a finding consistent with our study. These findings taken together suggest that factors other than acute infection with $C$. trachomatis are important in infertility. 
Both antibody to Chsp-60 and high IgG titers to C. trachomatis have been associated with both infertility and ectopic pregnancy. ${ }^{15,22}$ In our population, elevated Chsp-60 levels were related to tubal occlusion seen at the index episode of PID in our population, but not to a positive $G$. trachomatis culture. ${ }^{17}$ The relationship between tubal fertility and elevated titers of Chsp-60 and chlamydial IgG may result from several mechanisms. One mechanism is an exaggerated immune response. For example, in the present report, perhepatic adhesions were associated with infertility. During the acute episode of PID, perihepatic adhesions were associated with increased tubal damage seen and with Chsp- $60 .{ }^{23}$ Thus, perihepatic adhesions and tubal damage may not only result from acute infection but also from an immunopathologic response. The increased incidence and severity of fallopian tube inflammation caused by repetitive experimental infections with C. trachomatis are attributed to an exaggerated immune response. ${ }^{24}$ The presence of infertility among patients with an IgG antibody response could indicate a particularly large area (not only the tubes but also the peritoneal surface) of antigen surface stimulation, long standing infection with persistent chlamydial antigen, ${ }^{25}$ a genetic predisposition of certain HLA (human leukocyte antigen) types to an exaggerated immune response, ${ }^{26}$ or the exaggerated immune response from repetitive salpingitis. These factors and their importance have not been simultaneously examined together in an infertile population, and to do so, the population would have to be considerably larger than ours.

What can be made of the association between microorganisms and infertility? Infertility can be viewed as an end result of a complex pathogenetic process comprised of three components: infection, inflammation, and tissue repair. The high prevalence of infertility in our study probably resulted from the combined effect of infection in the index as well as prior and subsequent episodes of PID. The weak association of Chsp-60 and tubal infertility is consistent with the ability of repetitive infection to produce increased amounts of inflammation and, in turn, fibrosis. Prevention of recurrent PID and better understanding of the pathophysiology of postinfectious tubal damage (which probably differs between chlamydia and gonorrhea) is needed to develop more effective strategies to reduce permanent tubal damage.
Fibrosis can also lead to chronic pain. It is of interest that of the three patients with chronic pain, two patients had chronic pain prior to the index episode of PID. The $6 \%$ rate of chronic pain in this report is less than the $15 \%$ rate of chronic pain reported among women treated for PID in the 1960s and 1970s. ${ }^{27}$

We did not examine our infertile patients or confirm their infertility with HSG or laparoscopy, but Weström found tubal factor infertility in almost $90 \%$ of cases of infertile patients with PID who were subsequently examined. ${ }^{1,2}$ The majority (74\%) of our infertile patients had demonstrated reproductive and tubal function by a pregnancy prior to the index episode of PID. The strengths of the study were the confirmation of PID using laparoscopy and endometrial biopsy and the prospective ascertainment of demographic and reproductive history, physical findings, and microbiology. The weakness of the study was related to the limited number of women who could be located, despite major effort, 2 to 9 years after the index episode, which allowed us to identify variables only highly associated with infertility and potentially increases selection bias.

\section{REFERENCES}

1. Weström L. Effect of acute pelvic inflammatory disease on fertility. Am J Obstet Gynecol 1975;121:707-713.

2. Weström L, Joesoef MR, Reynolds GH, Hagdu A, Thompson SE. Pelvic inflammatory disease and fertility. Sex Transm Dis 1992;14:185-192.

3. Weström L. Incidence, prevalence, and trends of acute pelvic inflammatory disease and its consequences in industrialized countries. Am J Obstet Gynecol 1980;138: 880-892.

4. Cates W Jr, Wasserheit JN. Genital chlamydial infections: epidemiology and reproductive sequelae. Am J Obstet Gynecol 1991;164:1771-1781.

5. Jones RB, Ardery BR, Hui SC, Cleary RE. Correlation between serum antichlamydial antibodies and tubal factor as a cause of infertility. Fertil Steril 1982;38:553-558.

6. Moore DE, Spadoni LR, Foy HM, et al. Increased frequency of serum antibodies to Chlamydia trachomatis in infertility due to distal tubal disease. Lancet 1982;2(8298):574-577.

7. Miettinen A, Heinonen PK, Teisala K, Hakkarainen K, Punnonen R. Serologic evidence for the role of Chlamydia trachomatis, Neisseria gonorrhoeae, and Mycoplasma hominis in the etiology of tubal factor infertility and ectopic pregnancy. Sex Transm Dis 1990;17:10-14.

8. World Health Organization Task Force in the Prevention and Management of Infertility. Tubal infertility: 
serologic relationship to past chlamydial and gonococcal infection. Sex Transm Dis 1995;22:71-77.

9. Brunham RG, Binns B, Guijon F, et al. Etiology and outcome of acute pelvic inflammatory disease. J Infect Dis $1985 ; 158: 510-517$.

10. Svensson L, Mårdh P-A, Weström L. Infertility after acute salpingitis with special reference to Chlamydia trachomatis. Fertil Steril 1983;40:322-329.

11. Paavonen JA, Vesterinen E, Aantaa K, Rasanen J. Factors predicting abnormal hysterosalpingographic findings in patients treated for acute pelvic inflammatory disease. Int J Gynaecol Obstet 1985;23:172-175.

12. Jacobson L, Weström L. Objectivized diagnosis of acute pelvic inflammatory disease: diagnostic and prognostic value of routine laparoscopy. Am J Obstet Gynecol 1969;105:1088-1098.

13. Kiviat NB, Wölner-Hanssen P, Eschenbach DA, et al. Endometrial histopathology in patients with cultureproved upper genital tract infections and laparoscopically diagnosed acute salpingitis. Am J Surg Pathol 1990; 14:167-175.

14. Wasserheit JN, Bell TA, Kiviat NB, et al. Microbial causes of proven pelvic inflammatory disease and efficacy of clindamycin and tobramycin. Ann Intern Med 1986;104:187-193.

15. Toye B, Laferriere C, Glaman P, Jessamine P, Peeling R. Association between antibody to the Chlamydia heat shock protein and tubal infertility. J Infect Dis 1993; 168:1236-1240.

16. Wilcoxon F, Katti SK, Wilcox RA. Selected Tables in Mathematical Statistics. Providence, RI: American Mathematical Society, 1972. p 171-259.

17. Eckert LO, Hawes SE, Wolner-Hanssen P, et al. Prevalence and correlates of antibody to chlamydial heat shock protein in women STD clinic attendees and women confirmed PID. J Infect Dis 1997;175:14531458.

18. Soper D, Ness RB. Pelvic inflammatory disease and in- voluntary infertility: prospective pilot observations. Infect Dis Obstet Gynecol 1995;3:145-148.

19. Safrin S, Schachter J, Dahrouge D, Sweet RL. Longterm sequelae of acute pelvic inflammatory disease. A retrospective cohort study. Am J Obstet Gynecol 1992; 166:1300-1305.

20. Soper DE, Brockwell NJ, Dalton HP. Microbial etiology of urban emergency department acute salpingitis: treatment with ofloxacin. Am J Obstet Gynecol 1992;167: 653-660.

21. Brihmer G, Kallings I, Nord CE, Brundin J. Second look laparoscopy; evaluation of two different antibiotic regimens after treatment of acute salpingitis. Eur J Obstet Gynecol Reprod Biol 1989;30:263-274.

22. Brunham RC, Peeling R, Maclean I, Kosseim ML, Parastevas. Chlamydia trachomatis-associated ectopic pregnancy: serologic and histologic correlates. J Infect Dis 1992;165:1076-1081.

23. Money DH, Hawes SE, Eschenbach DA, et al. Antibodies to the chlamydial $60 \mathrm{kDa}$ heat shock protein are associated with laparoscopically confirmed perihepatitis. Am J Obstet Gynecol 1997;176:870-877.

24. Patton DL, Kuo C-C, Wang S, Halbert S.: Distal tubal obstruction induced by repeated Chlamydia trachomatis salpingeal infections in pig-tailed Macaques. J Infect Dis 1987;155:1292-1299.

25. Patton DL, Askienazy-Elbhar M, Henry-Suchet J, et al Detection of Chlamydia Trachomatis in fallopian tube tissue in women with post-infectious tubal infertility. Am J Obstet Gynecol 1994;171:95-101.

26. Kimani J, Maclean I, Bivay J, et al. Risk factors fo Chlamydia trachomatis pelvic inflammatory disease among sex workers in Nairobi, Kenya. J Infect Dis 1996; 173:1437-1444.

27. Falk, V. Treatment of acute non-tuberculosis salpingitis with antibiotics alone and in combination with glucocorticoids. Acta Obstet Gynecol Scand (Suppl.) 1965;44: $1-118$. 


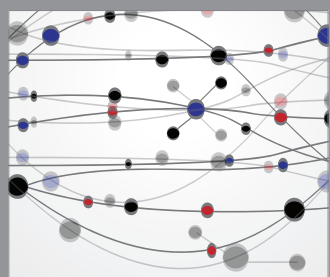

The Scientific World Journal
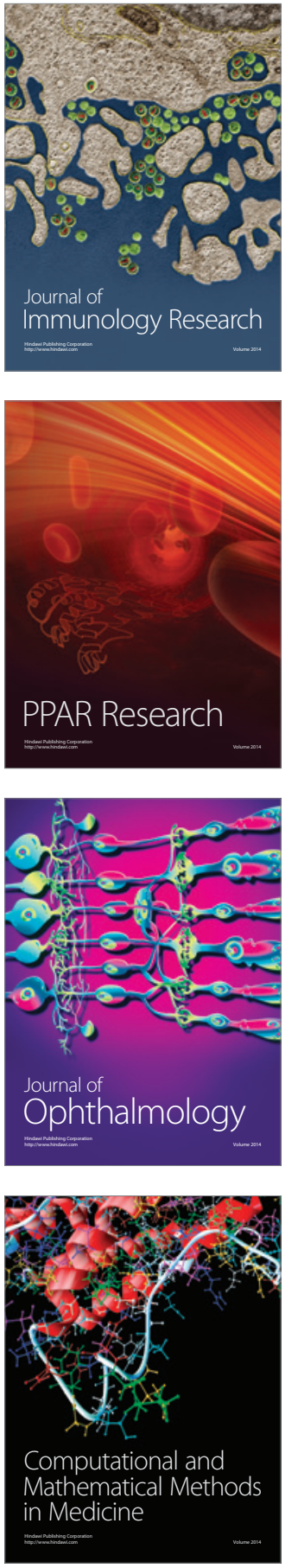

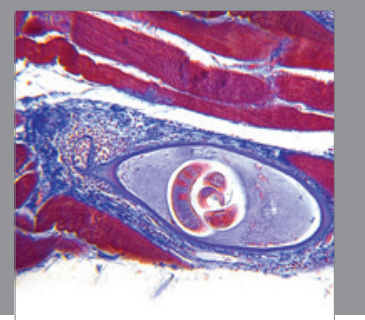

Gastroenterology

Research and Practice
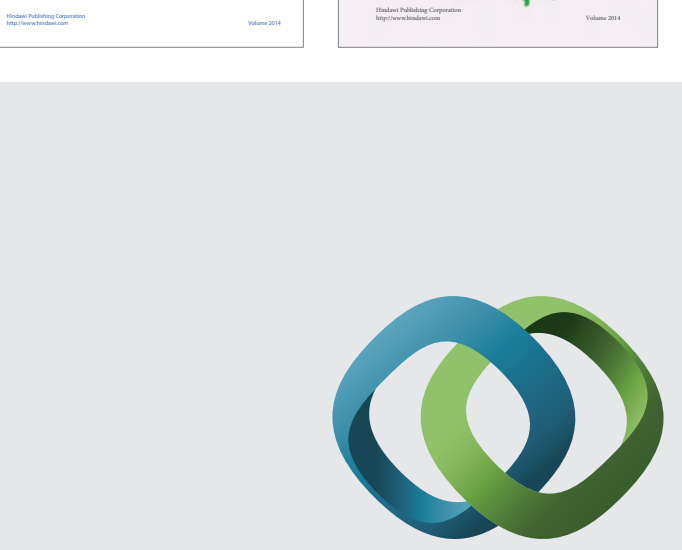

\section{Hindawi}

Submit your manuscripts at

http://www.hindawi.com
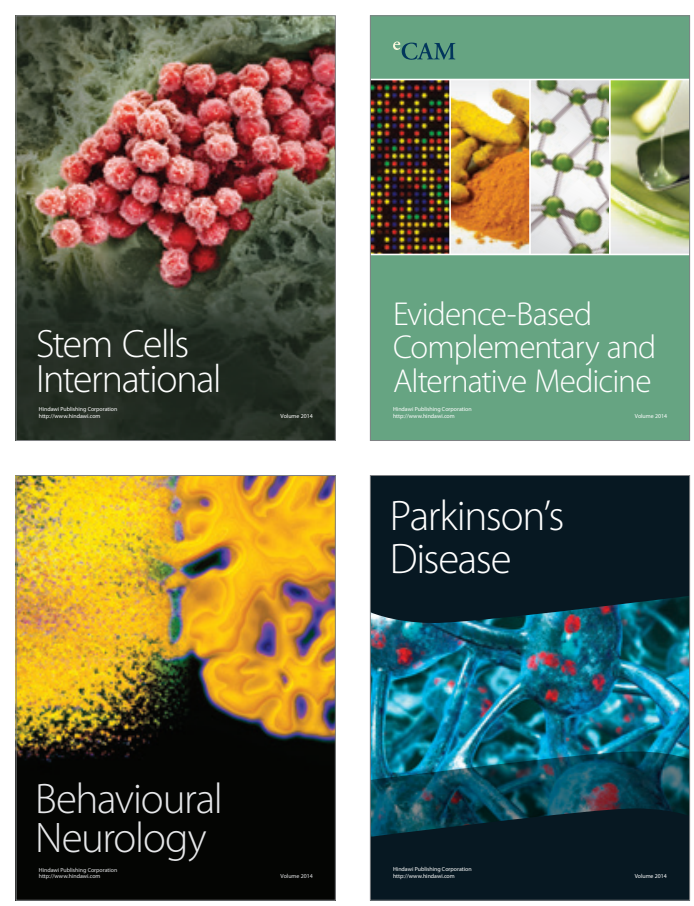

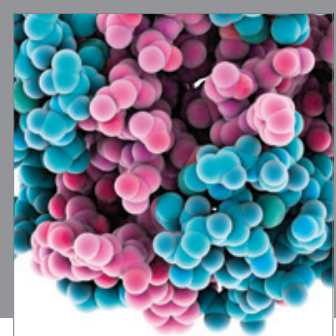

Journal of
Diabetes Research

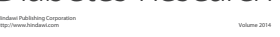

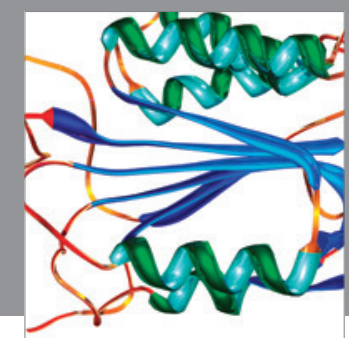

Disease Markers
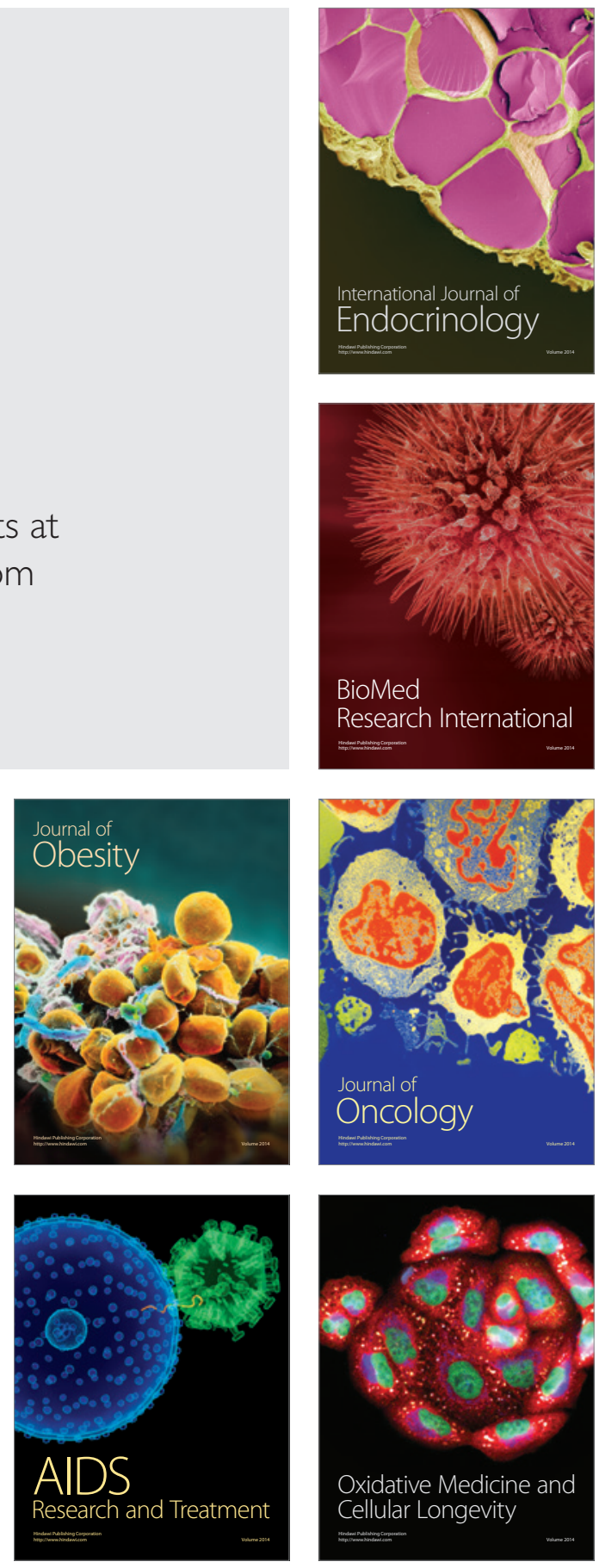